\title{
Performance Analysis of UAV-Assisted Wireless Powered Sensor Network over Shadowed $\kappa-\mu$ Fading Channels
}

\author{
Ivan Milovanovic $\mathbb{D D}^{1}$ and Caslav Stefanovic $\left.{ }^{2}\right)^{2}$ \\ ${ }^{1}$ Singidunum University, Danijelova 32, 11000 Belgrade, Serbia \\ ${ }^{2}$ Department of Signal Theory and Communications, Universidad Carlos III de Madrid, 28911 Leganes, Spain \\ Correspondence should be addressed to Caslav Stefanovic; caslav.stefanovic@uc3m.es
}

Received 9 March 2021; Accepted 28 March 2021; Published 22 April 2021

Academic Editor: Stefan Panic

Copyright (C) 2021 Ivan Milovanovic and Caslav Stefanovic. This is an open access article distributed under the Creative Commons Attribution License, which permits unrestricted use, distribution, and reproduction in any medium, provided the original work is properly cited.

\begin{abstract}
In this work, we analyze performances of the unmanned aerial vehicle- (UAV-) assisted wireless powered sensor communication, where sensor transmission ability is supported by the UAV. Harvested energy from the UAV-broadcasted signal is further used at the sensor nodes for uplink information transmission to the UAV, over assumed shadowed $\kappa-\mu$ fading channels. Here, we observe a general scenario in which due to the flight conditions of the UAV, the channel's content include the LOS components affected by the shadowing effect, modeled by the general shadowed $\kappa-\mu$ channel model, which can be reduced to other well-known channel models as its special cases. We derive closed-form expressions for the outage probability (OP) of such wireless sensor network (WSN) operating in shadowed $\kappa-\mu$ fading environments. Further, we analyze the optimization of time allocation to minimize OP subjected to UAV's energy constraints. The impact of channel parameters on observed performance measures is analyzed, and obtained results are numerically validated.
\end{abstract}

\section{Introduction}

The potential use of unmanned aerial vehicles (UAVs are also known as drones) for data collection in wireless sensor networks (WSNs) has procured significant interest recently due to their effectiveness and deployment flexibility with changing trajectory, which can be considered major advantages of UAV-assisted WSNs. Namely, in emergency situations, intelligent heterogeneous architectures based on UAV-assisted WSNs could be considered a promising new solution for developing future $5 \mathrm{G}$ communication networks based on ultrareliable low-latency communication (URLLC) and massive machine-type communications (mMTC). Various numbers of applications have been studied in the literature, for instance, mobile base stations $[1,2]$ and mobile relays in cooperative communication scenarios [3-5]. In [1], the authors have studied the optimal deployment of multiple UAVs equipped with directional antennas used as aerial base stations. The results obtained in [1] have shown that the number of available UAVs, the antenna gain, and beam width can determine the UAV optimal altitude and the loca- tion. A new polynomial time successive mobile base station placement solution for UAV ground terminals has been proposed in [2]. A new mobile relaying technique with high-mobility UAV relays to maximize throughput has been introduced in [3].

WSNs usually consist of a large number of powerconstraint low-cost sensor nodes (SNs). They are typically powered using rechargeable batteries and SNs are difficult to be recharged after depletion [6]. Considerable interest has been devoted to radio frequency (RF) energy harvesting (EH) techniques, i.e., wireless power transfer (WPT) and simultaneous wireless information and power transfer (SWIPT), to prolong battery life of power-constraint wireless SNs. Recent advances and challenges in RF-EH have been extensively studied in [7]. The outage performance of an $\mathrm{EH}$-enabled UAV-based wireless relay system has been studied in [4]. A new unified energy harvesting scheme for fullduplex UAV to assist a cooperative communication system is proposed in [5] to improve the operational time of the UAV while maintaining the expected quality-of-service (QoS). A UAV with WPT capability to collect sensor 
observations has been investigated in [8]. The authors exploited the mobility of the UAV to maximize the energy transfer to all SNs by optimizing the UAV trajectory. A new framework to improve energy efficiency in deadlinebased WSN data collection with multiple UAVs has been proposed in [9]. A novel data gathering framework in the UAV-assisted WSNs has been developed in [10]. The authors have introduced a priority-based data access scheme considering the mobility of the UAV to increase data gathering efficiency of the system. Flight time minimization of UAV for data collection over WSNs has been investigated in [11]. The authors have formulated the flying time minimization problem as a dynamic programming problem and solved the optimization problem in a one-dimensional sensor network.

In this work, we consider a UAV-assisted WSN, in which all SNs located within the UAV's coverage area are powered by the UAV using the WPT EH technique. Recharging or powering up SNs through WPT by the assistance of a data collecting UAV is a promising approach, especially when the SNs are located in a hazardous location or manual replacement of SNs batteries is challenging. Unlike in the existing literature, we emphasize fading channels between the UAV and SNs and explore the effect on efficient power transfer and data collection in the presence of fading channels. Assuming that the ground-to-air channels are experiencing shadowed $\kappa-\mu$ fading, we derive outage probability and obtain the optimal time ratio to minimize the outage probability numerically. To the best of the knowledge of the authors, there are no prior works conducted on $\mathrm{UAV}$-assisted wireless powered WSNs over shadowed $\kappa-\mu$ fading channels.

Presented model covers propagation scenarios with the following physical interpretation: multipath fading effects, wireless signal in a line-of-sight (LOS) environment while shadowing is considered to affect only the power of the dominant components. This general fading model, written in the function of three parameters: shadowing fluctuation parameter $m$; clusters parameter $\mu$; and dominant components, power/scattered wave power ratio parameter $\kappa$, can be simplified and transformed in other well-known fading models, by setting corresponding parameter values (i.e., one-sided Gaussian model: $\mu=0.5, \kappa \rightarrow 0, m \rightarrow 1$; Rayleigh model $\mu=$ $1, \kappa \rightarrow 0, m \rightarrow 1$; Nakagami- $m$ model, $\mu=m, \kappa \rightarrow 0, m \rightarrow 1$; Rician model, $\mu=1, \kappa=K, m \rightarrow 1 ; \kappa-\mu$ model, $\mu=\mu, \kappa=\kappa$, $m \rightarrow 1$; and Rician shadowed model, $\mu=1, \kappa=K, m=m$ ).

The remainder of this paper is organized as follows. Section 2 presents the detailed description of the system model and the time-switching (TS) protocol used in this paper. System performance analysis including the analytical expression of the outage probability and optimal time ratio is presented in Section 3. In Section 4, numerical results are presented. Finally, Section 5 concludes the paper.

\section{System Model}

We consider a UAV-assisted WSN as illustrated in Figure 1, where a UAV collects sensor observation in a circular service area of radius $R$. The UAV is hovering at the center of the cir-

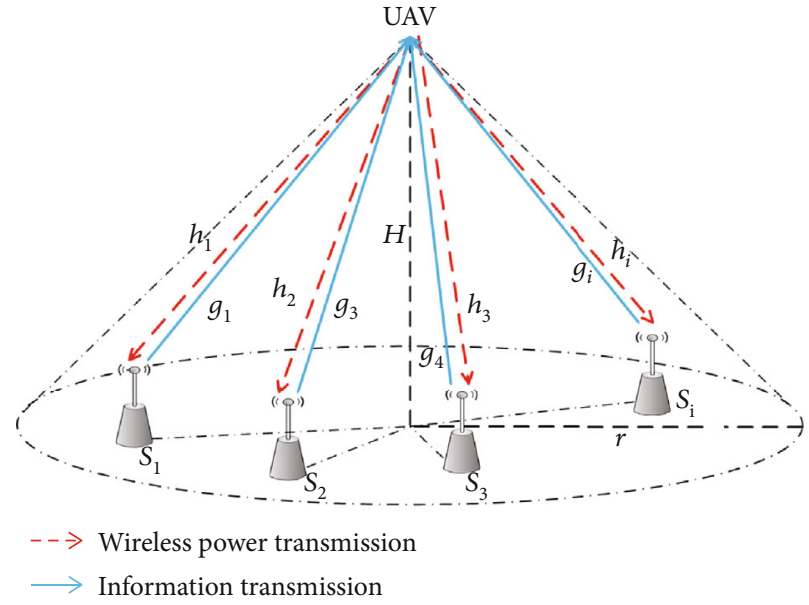

Figure 1: A reference system model for wireless powered wireless sensor network with energy transfer in downlink and information transmission in the uplink.

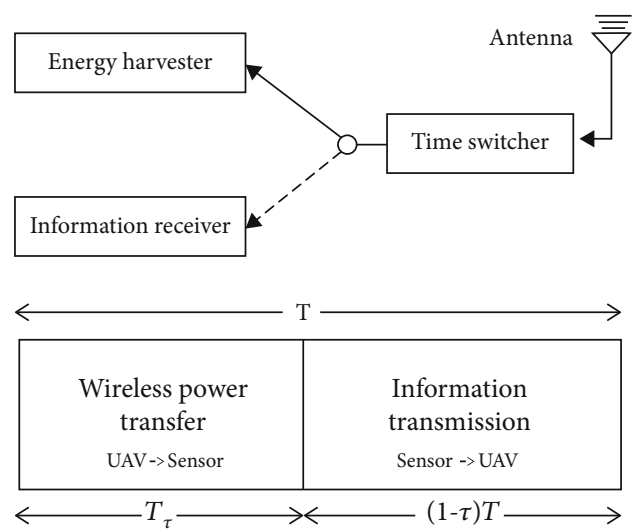

FIgURE 2: Transmission block diagram of the proposed system.

cular service area with the height of $H$. We assume that the $k$ number of SNs is randomly located within the UAV's coverage area. In addition, all SNs and the UAV are equipped with a single antenna. Further, it is assumed that the SNs do not have any prior power supply or charge in its inbuilt batteries for information transmission, while the UAV is equipped with two batteries. One battery of the UAV is used for its mobility, while the other will be used as a constant power source with the transmission power $P_{0}$ that is exclusively used for the WPT.

Once UAV reaches the center of the service area, the data collection process starts and the total operational data collecting time denotes by $T$. The TS protocol structure is used in the system as shown in Figure 2. During the first time phase $T \tau$, where $\tau \in(0,1)$, the UAV will broadcast the WPT signal to power-up the SNs. At the remaining time fraction, $T(1-\tau)$ is used by the SNs to send sensor observations to the UAV. We adopt $i \in\{1,2, \cdots, N\}$ as the index of the SNs. Let $h_{i}=h_{u s_{i}}$ and $g_{i}=h_{s, u}$ denote the channel coefficients from the UAV to the $i$ th SN and $i$ th SN to UAV, respectively. Furthermore, $h_{u s_{i}}$ and $h_{s_{i} u}$ are assumed to be independent for all SNs. Location of the $i$ th $\mathrm{SN}$ is denoted by $\left(r_{i}, \phi_{i}\right)$ in the 
polar coordinates on the ground plane. Thus, the squared Euclidean distance between the UAV and the $i$ th $\mathrm{SN}$ can be written as $d_{i}^{2}=H^{2}+r_{i}^{2}$, where $r_{i}$ is the distance from the $i$ th $\mathrm{SN}$ to the center of the UAV service area. Let $w_{i}$ denote the path loss between the $i$ th $\mathrm{SN}$ and UAV, which can be expressed as $w_{i}^{2}=K_{i}^{2 \alpha}$, where $\alpha$ is the path loss exponent and $K_{i}$ denotes the constant coefficient of the path loss. In addition, we assume that all the SNs attempt to transmit at a constant information transmission rate denoted by $R$ per channel use. There is no interference among the SNs during the second time fraction of the TS protocol due to the fact that all SNs are assigned to different orthogonal channels. Accordingly, the amount of energy harvested by each SN during the first time phase in the TS protocol is given as

$$
E_{i}=\eta\left|h_{u s_{i}}\right|^{2} w_{i} P_{0} \tau T
$$

where $\eta$ is EH efficiency of the EH circuitry in SN. Thus, the transmit power of the $i$ th $\mathrm{SN}$ can be calculated as

$$
P_{s_{i}}=\frac{E_{i}}{(1-\tau) T}=\eta \frac{\tau}{1-\tau}\left|h_{u s_{i}}\right|^{2} w_{i} P_{0}
$$

Then, the SNR of the signal received by the UAV from the $i$ th $\mathrm{SN}$ can be expressed as

$$
\gamma_{u_{i}}=\left|h_{s_{i} u}\right|^{2}\left|h_{u s_{i}}\right|^{2} w_{i}^{2} \eta \frac{\tau}{1-\tau} \frac{P_{0}}{N_{0 i}},
$$

where $N_{0 i}$ is the power of additive Gaussian white noise (AWGN) between the $i$ th $\mathrm{SN}$ and the UAV. Finally, the achievable information rate for sensor observations of the $i$ th $\mathrm{SN}$ can be written as $C_{i}=(1-\tau) \log _{2}\left(1+\gamma_{u_{i}}\right)$. Due to the flying altitude of the UAV, the channels do not contain a line-of-sight (LoS) component but are often modeled by shadowed $\kappa-\mu$ fading [12]. Thus, shadowed $\kappa-\mu$ PDF can be expressed as

$$
\begin{gathered}
f_{X_{i}}\left(X_{i}\right)=\frac{\mu_{i}^{\mu_{i}} m_{i}^{m_{i}}\left(1+\kappa_{i}\right)^{\mu_{i}}}{\Gamma\left(\mu_{i}\right)\left(\mu_{i} \kappa_{i}+m_{i}\right)} \exp \left(-\frac{\mu_{i}\left(1+\kappa_{i}\right) X_{i}}{\Omega_{i}}\right) \\
\frac{X_{i}^{\mu_{i}-1}}{\Omega_{i}^{m_{i}}} F_{1}\left(m_{i}, \mu_{i}, \frac{\mu_{i}^{2} \kappa_{i}\left(\kappa_{i}+1\right) X_{i}}{\left(\mu_{i} \kappa_{i}+m_{i}\right) \Omega_{i}}\right),
\end{gathered}
$$

where $\Omega_{i}$ denotes the channel average SNR value.

\section{Performance Analysis}

In this section, we investigate the system performance of the system model by deriving the mathematical expression for its outage probability.

3.1. Outage Probability. Under the system model constructed in Section 2 and given the targeted information rate $R$, the outage probability can be written as
TABLE 1: Observed values of system parameters.

\begin{tabular}{lc}
\hline System model parameters & Values \\
\hline UAV to ground distance & $h=500 \mathrm{~m}$ \\
Sensor's targeted data rate & $R=1 \mathrm{bit}$ \\
Coefficient of energy conversion & $\eta=0.9$ \\
Path loss exponent & $\alpha=3$ \\
Noise power & $N_{0}=-150 \mathrm{dBm}$ \\
Constant coefficient of path loss & $w_{i}=1.3$ \\
Radius of the UAV's serviced area & $r=100 \mathrm{~m}$ \\
\hline
\end{tabular}

$$
\begin{aligned}
\operatorname{Pr}\left(C_{i}<R\right) & =\operatorname{Pr}\left(\frac{\mathrm{R}}{1-\tau}>\log _{2}\left(1+\gamma_{u_{i}}\right)\right) \\
& =\operatorname{Pr}\left(\gamma_{u_{i}}<2^{\mathrm{R} /(1-\tau)}-1\right) \\
& =\operatorname{Pr}\left(\frac{\left|h_{s_{i} u}\right|^{2}\left|h_{u s_{i}}\right|^{2}}{\left(H^{2}+r_{i}^{2}\right)^{\alpha}} w_{i}^{2} \eta \frac{\tau}{1-\tau} \frac{P_{0}}{N_{0_{i}}}<\left(2^{\mathrm{R} /(1-\tau)}-1\right)\right) \\
& =\operatorname{Pr}\left(\frac{\left|h_{s_{i} u}\right|^{2}\left|h_{u s_{i}}\right|^{2}}{\left(H^{2}+r_{i}^{2}\right)^{\alpha}}<\mathscr{T}\left(\tau, R, \frac{P_{0}}{N_{0_{i}}}\right)\right),
\end{aligned}
$$

where $\mathscr{T}\left(\tau, R, P_{0} / N_{0_{i}}\right)=\left(2^{\mathrm{R} /(1-\tau)}-1\right)\left(1 / w_{i}^{2} \eta\right)((1-\tau) / \tau)\left(N_{0_{i}}\right.$ $\left./ P_{0}\right)$. For given $r_{i}$, the above equation can be written as

$\operatorname{Pr}\left(C_{i}<R \mid r_{i}\right)=\operatorname{Pr}\left(\left|h_{s_{i} u} h_{u s_{i}}\right|<\sqrt{\mathscr{T}\left(\tau, \mathrm{R}, P_{0} / N_{0_{i}}\right)\left(H^{2}+r_{i}^{2}\right)^{\alpha}}\right)$.

Thus, the distribution of $z_{i}=\left|h_{u s_{i}} h_{s_{i} u}\right|$ can be expressed as

$$
\begin{aligned}
& f_{z_{i}}(z)=\sum_{p=0}^{\infty} \sum_{s=0}^{\infty} \frac{2 \mu_{g}^{\left(\mu_{g}+\mu_{h}+3 s+p\right) / 2} \mu_{h}^{\left(\mu_{g}+\mu_{h}+s+3 p\right) / 2} m_{g}^{m_{g}} m_{h}^{m_{h}}}{\left(\mu_{g} \kappa_{g}+m_{g}\right)^{m_{g}+s}\left(\mu_{h} \kappa_{h}+m_{h}\right)^{m_{h}+p}} \\
& \times \frac{\kappa_{g}^{s} \kappa_{h}^{p}\left(\left(1+\kappa_{g}\right)\left(1+\kappa_{h}\right)\right)^{\left(\mu_{g}+\mu_{h}+s+p\right) / 2}}{\left(\Omega_{g} \Omega_{h}\right)^{\left(\mu_{g}+\mu_{h}+s+p\right) / 2} \Gamma\left(m_{g}\right) \Gamma\left(m_{h}\right)} \\
& \times \frac{\Gamma\left(m_{g}+s\right) \Gamma\left(m_{h}+p\right)}{\Gamma\left(\mu_{g}+s\right) \Gamma\left(\mu_{h}+p\right) p ! s !} z^{\left(\left(\mu_{g}+\mu_{h}+s+p\right) / 2\right)-1} \\
& \times K_{\left(\mu_{h}-\mu_{g}+p-s\right)}\left(2 \sqrt{\frac{\mu_{h} \mu_{g}\left(1+\kappa_{g}\right)\left(1+\kappa_{h}\right)}{\Omega_{g} \Omega_{h}} z}\right),
\end{aligned}
$$

where $g=h_{u s_{i}}$ and $h=h_{s_{i} u}$. The outage probability, $\operatorname{Pr}\left(z_{i}<\right.$ $\left.Z \mid r_{i}\right), \operatorname{Pr}\left(z_{i}<Z \mid r_{i}\right)=\int_{0}^{Z\left(r_{i}\right)} f_{z_{i}}(z) d z$ is shown on top of the next page, In practice, upon numerical evaluation of (8) the infinite series with respect to $i$ and 1 should be truncated. We have chosen the summation upper limit as 10 , which is found to be large enough such that the truncation error is negligible in our simulation setups, where 


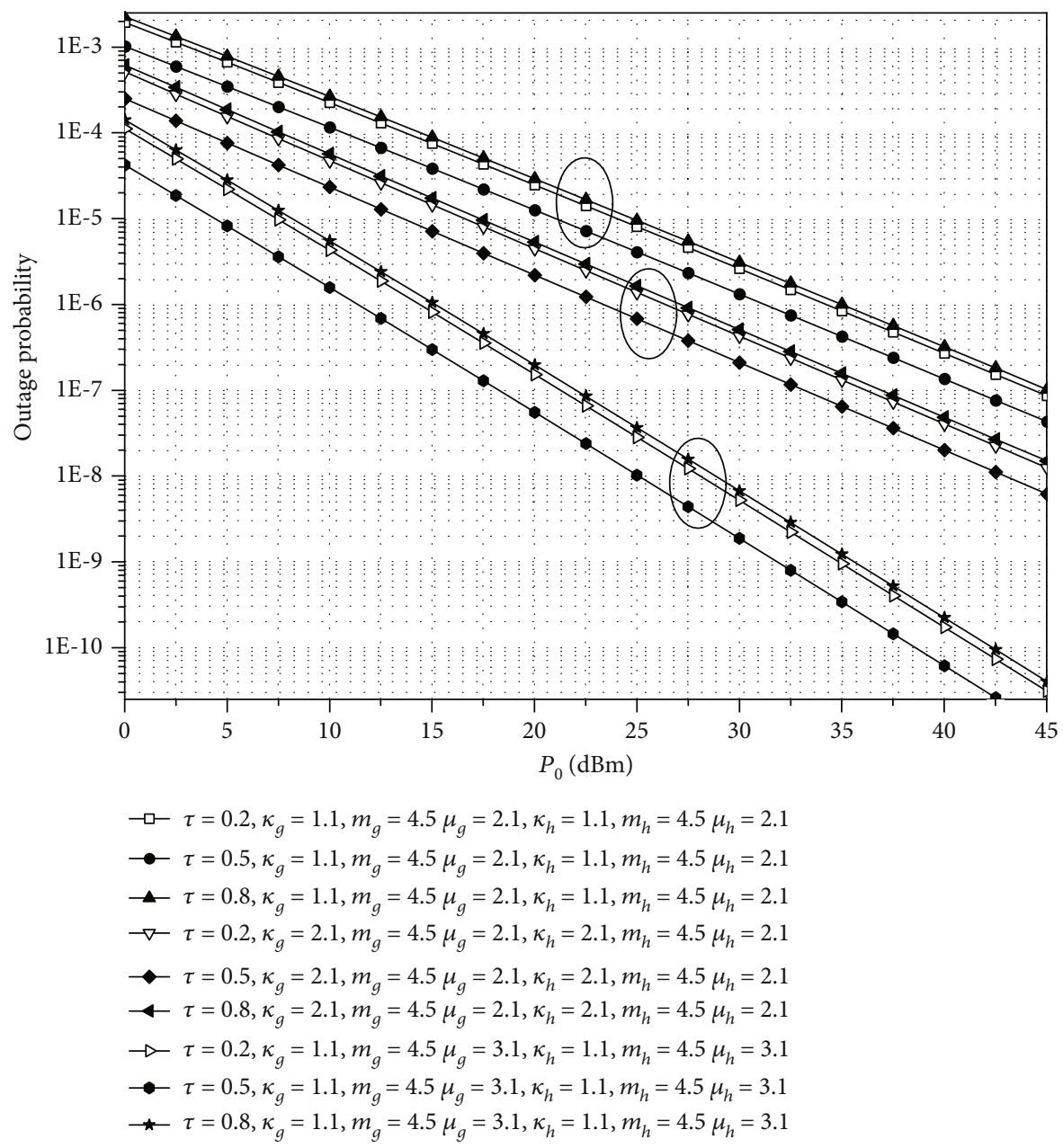

FIGURE 3: Outage probability versus the transmit power of UAV $P_{0}$.

$$
\begin{aligned}
\operatorname{Pr}\left(z_{i}<Z \mid r_{i}\right)= & \sum_{p=0}^{\infty} \sum_{s=0}^{\infty} \frac{\mu_{g}^{\left(\mu_{g}+\mu_{h}+3 s+p\right) / 2} \mu_{h}^{\left(\mu_{g}+\mu_{h}+s+3 p\right) / 2} m_{g}^{m_{g}} m_{h}^{m_{h}}}{\left(\mu_{g} \kappa_{g}+m_{g}\right)^{m_{g}+s}\left(\mu_{h} \kappa_{h}+m_{h}\right)^{m_{h}+p}} \\
& \cdot \frac{\kappa_{g}^{s} \kappa_{h}^{p}\left(\left(1+\kappa_{g}\right)\left(1+\kappa_{h}\right)\right)\left(\mu_{g}+\mu_{h}+s+p\right) / 2}{\left(\Omega_{g} \Omega_{h}\right)^{\left(\mu_{g}+\mu_{h}+s+p\right) / 2} \Gamma\left(m_{g}\right) \Gamma\left(m_{h}\right)} \\
& \times \frac{\Gamma\left(m_{g}+s\right) \Gamma\left(m_{h}+p\right)}{\Gamma\left(\mu_{g}+s\right) \Gamma\left(\mu_{h}+p\right) p ! s !}\left(Z\left(r_{i}\right)\right)^{\left(\mu_{g}+\mu_{h}+s+p\right) / 2} G_{1,3}^{2,1} \\
& \cdot\left(B Z\left(r_{i}\right) \mid \begin{array}{l}
1-\frac{\mu_{g}+\mu_{h}+s+p}{2}, \\
\frac{\mu_{h}-\mu_{h}+p-s}{2},-\frac{\mu_{g}-\mu_{h}+s-p}{2},-\frac{\mu_{g}+\mu_{h}+s+p}{2},
\end{array}\right) .
\end{aligned}
$$

$$
\begin{gathered}
B=\frac{\mu_{h} \mu_{g}\left(1+\kappa_{g}\right)\left(1+\kappa_{h}\right)}{\Omega_{g} \Omega_{h}}, \\
Z\left(r_{i}\right)=\sqrt{\mathscr{T}\left(\alpha, \mathrm{R}, P_{0} / N_{0_{i}}\right)\left(H^{2}+r_{i}^{2}\right)^{\alpha}} .
\end{gathered}
$$

The proof is provided in the appendix.

3.2. Time-Switching Factor Optimization. Here, we try to optimize the value for $\tau$, which minimizes the outage probability. As we can observe from (6), when the SN location $r_{i}$ is fixed, outage probability minimization is equivalent to $\mathscr{T}(\tau$ , $\left.R, P_{0} / N_{0_{i}}\right)$ in (5). Therefore, for the given values for $P_{0}$, $N_{0_{i}}$, and rate $R$, by selecting a value for $\tau \in\{0,1\}$ outage probability can be minimized. To satisfy the local optimality, partial derivatives of $\mathscr{T}\left(\tau, R, P_{0} / N_{0_{i}}\right)$ with respect to $\tau$ should be equal to zero. Thus, adhering to the local optimal condition, we can represent the partial derivatives of $\mathscr{T}\left(\tau, R, P_{0} /\right.$ $N_{0_{i}}$ ) with respect to $\tau$ as follows:

$$
\frac{\partial \mathscr{T}\left(\tau, R, P_{0} / N_{0_{i}}\right)}{\partial \tau}=0 .
$$

As it can be observed from (8), $\tau^{*}$ depends on the value of rate $R$, which involves joint computations of $\tau$ and rate $R$ making it a challenging to obtain as an exact solution and cannot be expressed by an explicit equation with respect to rate $R$. Therefore, we have obtained $\tau^{*}$ numerically in Section 4 .

\section{Numerical Results}

Here, we provide numerical results to demonstrate and validate the theoretical analysis presented in Sections 2 and 3. 


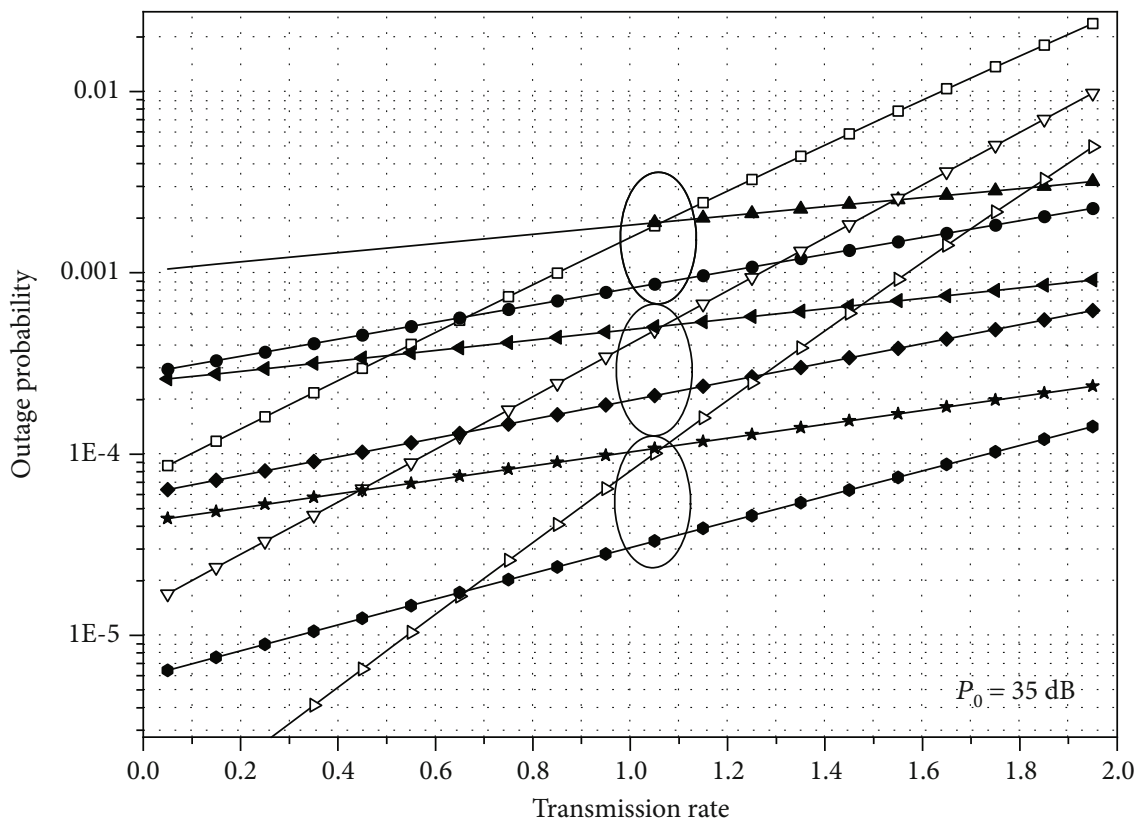

$\begin{aligned} \rightarrow \tau & =0.2, \kappa_{g}=1.1, m_{g}=4.5 \mu_{g}=2.1, \kappa_{h}=1.1, m_{h}=4.5 \mu_{h}=2.1 \\ \longrightarrow & =0.5, \kappa_{g}=1.1, m_{g}=4.5 \mu_{g}=2.1, \kappa_{h}=1.1, m_{h}=4.5 \mu_{h}=2.1 \\ \neg \tau & =0.8, \kappa_{g}=1.1, m_{g}=4.5 \mu_{g}=2.1, \kappa_{h}=1.1, m_{h}=4.5 \mu_{h}=2.1 \\ \rightarrow-\tau & =0.2, \kappa_{g}=2.1, m_{g}=4.5 \mu_{g}=2.1, \kappa_{h}=2.1, m_{h}=4.5 \mu_{h}=2.1 \\ \bullet \tau & =0.5, \kappa_{g}=2.1, m_{g}=4.5 \mu_{g}=2.1, \kappa_{h}=2.1, m_{h}=4.5 \mu_{h}=2.1 \\ \neg \tau & =0.8, \kappa_{g}=2.1, m_{g}=4.5 \mu_{g}=2.1, \kappa_{h}=2.1, m_{h}=4.5 \mu_{h}=2.1 \\ \rightarrow \tau & =0.2, \kappa_{g}=1.1, m_{g}=4.5 \mu_{g}=3.1, \kappa_{h}=1.1, m_{h}=4.5 \mu_{h}=3.1 \\ \rightarrow-\tau & =0.5, \kappa_{g}=1.1, m_{g}=4.5 \mu_{g}=3.1, \kappa_{h}=1.1, m_{h}=4.5 \mu_{h}=3.1 \\ \star \tau & =0.8, \kappa_{g}=1.1, m_{g}=4.5 \mu_{g}=3.1, \kappa_{h}=1.1, m_{h}=4.5 \mu_{h}=3.1\end{aligned}$

FIGURE 4: Outage probability versus information rate $R$.

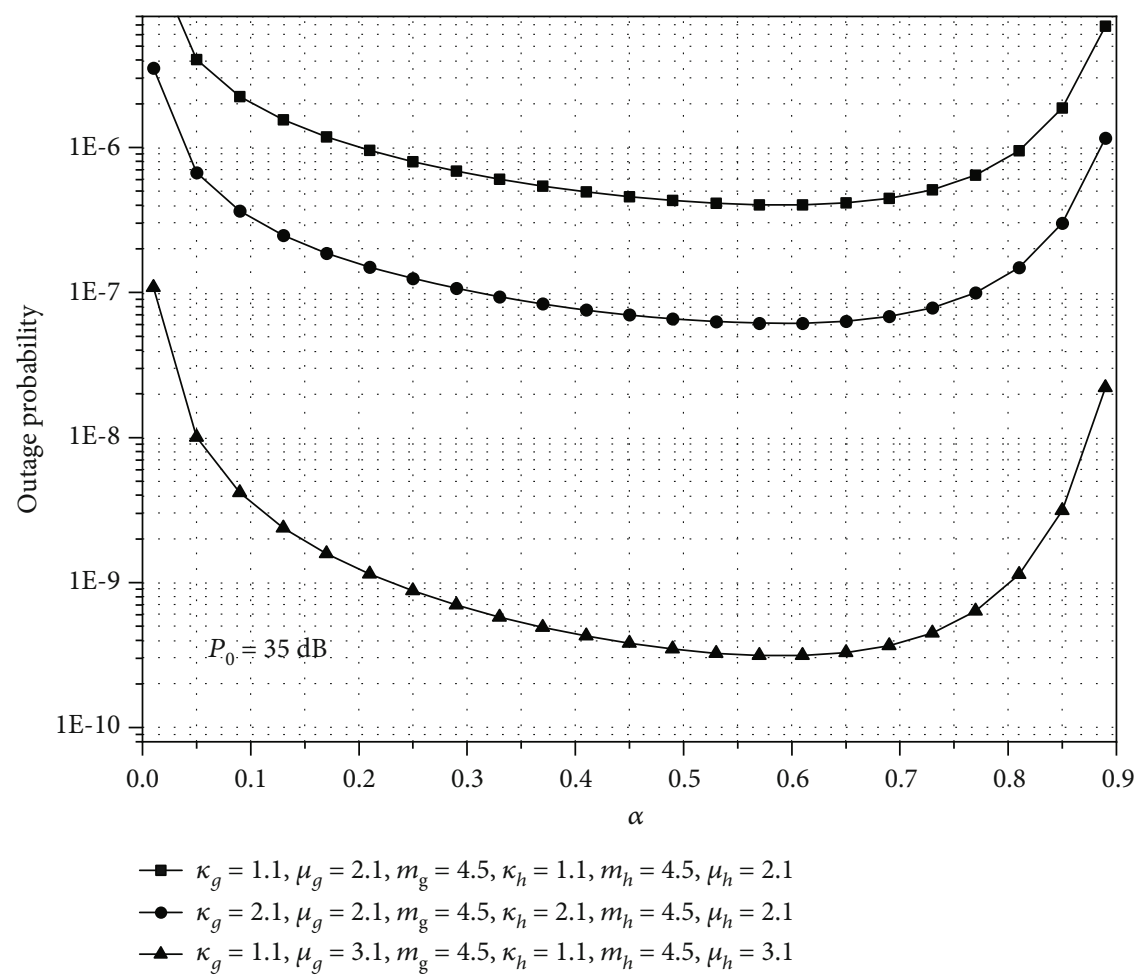

Figure 5: Outage probability versus the time ratio $\tau$ in the function of shadowed $\kappa-m u$ fading severity parameter. 
Unless otherwise stated, in all our simulations, we have used the parameters listed in Table 1.

In Figure 3, we observe OP values with respect to transmission power of the UAV $P_{0}$ for different $\tau$ ratio values, where $\tau \in\{0.2,0.5,0.8\}$. It can also be observed from Figure 3 that the increase in $\tau$ ratio values, first decrease the $\mathrm{OP}$ values and then increase them as expected. This is due to the fact that the increase in $\tau$ leads to reduction of transmission time, which eventually increases the overall OP of the system. It is interested to observe that, as expected, OP values decrease for higher values of parameters $\kappa_{g}, \kappa_{h}, m_{g}$, and $m_{h}$, while impact of $\kappa_{g}$ and $\kappa_{h}$ is more notable for higher values of $\mu_{g}$ and $\mu_{h}$.

Next, we observe OP versus transmission rate $R$ in Figure 4 with respect to different values of $\tau$ and fading channel parameters $\kappa_{g}, \kappa_{h}, \mu_{g}$ and $\mu_{h}, m_{g}$, and $m_{h}$. As it can be observed from Figure 4 , nevertheless of the change of the $\tau$ value, OP increases with the increase in information transmission rate. This observation also clearly depicts compromise between the information rate and resulting OP. In addition, Figure 4 shows that the optimal value of $\tau^{*}$ is changing based on the change of information transmission rate values. It is interesting to note that the sensors need more time to transmit data in order to minimize the OP, which can lead to a decrease in the value of $\tau^{*}$.

In order to find the optimal $\tau$ value, which minimizes OP, we plot the OP versus $\tau$, where $0<\tau<1$ as illustrated in Figure 5. Figure 5 shows that the outage probability decreases as $\tau$ increases from 0 to optimal $\tau^{*}(\approx 0.6)$ but later, it starts increasing as $\tau$ increases from its optimal value. This is due to the fact that for the values of $\tau$ smaller than the optimal value $\tau^{*}$, there is less time allocated for EH. Hence, less energy is harvested and larger values for outage probability can be observed due to smaller SN's transmission power (see (6)). On the other hand, for $\tau$ values greater than $\tau^{*}$, less time is available for information transmission. As a result, again outage probability increases at the UAV due to smaller value $(1-\tau) / 2$ (see Figure 2). Furthermore, it can be also observed from Figure 5 that an increase in shadowed $\kappa-\mu$ fading parameters $\kappa_{g}$ and $\kappa_{h}$ decreases the OP.

\section{Conclusion}

In this paper, we have analyzed a UAV-assisted WSN over shadowed $\kappa-\mu$ fading channels. We derived rapidly converging closed-form expressions for the OP of the observed system. Capitalizing on these expressions, OP has been discussed in the function of transmission system parameters. Furthermore, based on obtained expressions, we have observed the optimal time allocation, which minimizes the $\mathrm{OP}$. The results suggest that there is a compromise between the OP and transmission rate, so an optimal time ratio could be obtained depending on the required information rate. Finally, we identify the achievable OP determined by the calculated optimal time ratio based on the required transmission rate.

\section{Appendix}

In order to obtain PDF of $z_{i}=\left|h_{s_{i} u}\right|\left|h_{u s_{i}}\right|$, we must perform the following transformation of random variables:

$$
f_{z_{i}}(z)=\int_{0}^{\infty} f_{\left|h_{s_{i} u}\right|}\left(\frac{z_{i}}{\left|h_{u s_{i}}\right|}\right) f_{\left|h_{u s_{i}}\right|}\left(\left|h_{u s_{i}}\right|\right) \frac{1}{\left|h_{u s_{i}}\right|} d\left|h_{u s_{i}}\right|,
$$

where $\left|h_{s_{i} u}\right|$ and $\left|h_{u s_{i}}\right|$ have the PDFs given with Equation (7) and corresponding PDF parameters. After using series expressions of $1 \mathrm{~F} 1$ confluent hypergeometric functions and introducing into Equation (A.1), we obtained the following integral expression:

$$
\begin{aligned}
f_{z_{i}}(z)= & \sum_{p=0}^{\infty} \sum_{s=0}^{\infty} \frac{\mu_{g}^{2 s+\mu_{g}} \mu_{h}^{2 p+\mu_{h}} m_{g}^{m_{g}} m_{h}^{m_{h}} \kappa_{g}^{s} \kappa_{h}^{p}}{\left(\mu_{g} \kappa_{g}+m_{g}\right)^{m_{g}+s}\left(\mu_{h} \kappa_{h}+m_{h}\right)^{m_{h}+p}} \\
& \cdot \frac{\left(1+\kappa_{g}\right)^{\mu_{g}+s}\left(1+\kappa_{h}\right)^{\mu_{h}+p}}{\Omega_{g}^{\mu_{g}+s} \Omega_{h}^{\mu_{h}+p} \Gamma\left(m_{g}\right) \Gamma\left(m_{h}\right)} \frac{\Gamma\left(m_{g}+s\right) \Gamma\left(m_{h}+p\right)}{\Gamma\left(\mu_{g}+s\right) \Gamma\left(\mu_{h}+p\right) p ! s !} z^{\mu_{g}+s-1} \\
& \cdot \int_{0}^{\infty}\left|h_{u s_{i}}\right|^{\mu_{h}-\mu_{g}+p-s-1} \exp \left(-\frac{\mu_{g}\left(1+\kappa_{g}\right) z}{\Omega_{g}\left|h_{u s_{i}}\right|}\right) \times \exp \\
& \cdot\left(-\frac{\mu_{h}\left(1+\kappa_{h}\right)\left|h_{u s_{i}}\right|}{\Omega_{h}}\right) d\left|h_{u s_{i}}\right|
\end{aligned}
$$

which can be solved using Eq. (3.471.9) from [13]. In that way, we are obtaining the expression from Equation (7) expressed in the form of modified Bessel function of the second kind.

Now, by transforming the expression for modified Bessel function of the second kind into the expression for Meijer G function, by using Eq. (03.04.26.0008.01) from [14], we can obtain its CDF form expressed as Equation (8).

\section{Data Availability}

The authors confirm that the data supporting the findings of this study are available within the article and the appendix of the article. Moreover, the data that support the findings of this study are also available from the authors in the form of programme files, upon reasonable request.

\section{Conflicts of Interest}

The authors declare that they have no conflicts of interest.

\section{Acknowledgments}

Caslav Stefanovic would like to acknowledge CONEX-Plus programme. The CONEX-Plus is funded by UC3M, the European Commission through the Marie Sklodowska Curie COFUND Action (H2020-MSCA-COFUND-2017GA 801538). 


\section{References}

[1] M. Mozaffari, W. Saad, M. Bennis, and M. Debbah, "Efficient deployment of multiple unmanned aerial vehicles for optimal wireless coverage," IEEE Communications Letters, vol. 20, pp. 1647-1650, 2016.

[2] J. Lyu, Y. Zeng, R. Zhang, and T. J. Lim, "Placement optimization of uav-mounted mobile base stations," IEEE Communications Letters, vol. 21, pp. 604-607, 2017.

[3] Y. Zeng, R. Zhang, and T. J. Lim, “Throughput maximization for UAV-enabled mobile relaying systems," IEEE Transactions on Communications, vol. 64, pp. 4983-4996, 2016.

[4] L. Yang, J. Chen, M. O. Hasna, and H.-C. Yang, "Outage performance of UAV-assisted relaying systems with RF energy harvesting," IEEE Communications Letters, vol. 22, pp. 24712474, 2018.

[5] D. N. K. Jayakody, T. D. P. Perera, P. Muthuchidambaranathan, and M. O. Hasna, "Self-energized full duplex uav-assisted cooperative communication systems," in 2019 IEEE 7th Black Sea Conference on Communications and Networking. IEEE, Sochi, Russia, 2019..

[6] C. Zhan, Y. Zeng, and R. Zhang, "Energy-efficient data collection in UAV enabled wireless sensor network," IEEE Wireless Communications Letters, vol. 7, pp. 328-331, 2018.

[7] T. D. P. Perera, D. N. K. Jayakody, S. K. Sharma, S. Chatzinotas, and J. Li, "Simultaneous wireless information and power transfer (SWIPT): recent advances and future challenges," IEEE Communications Surveys \& Tutorials, vol. 20, pp. 264-302, 2017.

[8] J. Xu, Y. Zeng, and R. Zhang, "UAV-enabled wireless power transfer: trajectory design and energy optimization," IEEE Transactions on Wireless Communications, vol. 17, pp. 50925106, 2018.

[9] A. T. Albu-Salih and S. A. H. Seno, "Energy-efficient data gathering framework-based clustering via multiple uavs in deadline-based wsn applications," IEEE Access, vol. 6, pp. 72275-72286, 2018.

[10] S. Say, H. Inata, J. Liu, and S. Shimamoto, "Priority-based data gathering framework in uav-assisted wireless sensor networks," IEEE Sensors Journal, vol. 16, pp. 5785-5794, 2016.

[11] J. Gong, T. Chang, C. Shen, and X. Chen, "Flight time minimization of uav for data collection over wireless sensor networks," IEEE Journal on Selected Areas in Communications, vol. 36, pp. 1942-1954, 2018.

[12] J. Paris, "Statistical characterization of $\$ \backslash$ kappa $\{-\} \backslash \mathrm{mu} \$$ shadowed fading," IEEE Transactions on Vehicular Technology, vol. 63, pp. 518-526, 2014.

[13] I. S. Gradshteyn and I. M. Ryzhik, Table of Integrals, Series, and Products, Academic press, 2014.

[14] W. Research, "The mathematical functions site," http:// functions.wolfram.com/, 2021, [Online; accessed 2021]. 